\title{
Feasibility of concomitant cisplatin with hypofractionated radiotherapy for locally advanced head and neck squamous cell carcinoma
}

Alexandre Arthur Jacinto ${ }^{1 *}$ (D) Eronides Salustiano Batalha Filho ${ }^{1}$, Luciano de Souza Viana ${ }^{2}$, Pedro De Marchi ${ }^{2}$, Renato de Castro Capuzzo ${ }^{3}$, Ricardo Ribeiro Gama ${ }^{3}$, Domingos Boldrini Junior ${ }^{3}$, Carlos Roberto Santos ${ }^{3}$, Gustavo Dix Junqueira Pinto ${ }^{2}$, Josiane Mourão Dias ${ }^{2}$, Heloisa Pelisser Canton ${ }^{1}$, Raiany Carvalho ${ }^{3}$, Lucas Augusto Radicchi ${ }^{1}$, Soren Bentzen ${ }^{5}$, Eduardo Zubizarreta ${ }^{4}$ and Andre Lopes Carvalho ${ }^{3}$

\begin{abstract}
Background: The evolution of radiotherapy over recent decades has reintroduced the hypofractionation for many tumor sites with similar outcomes to those of conventional fractionated radiotherapy. The use of hypofractionation in locally advanced head and neck cancer (LAHNC) has been already used, however, its use has been restricted to only a few countries. The aim of this trial was to evaluate the safety and feasibility of moderate hypofractionated radiotherapy (HYP-RT) with concomitant cisplatin (CDDP).

Methods: This single-arm trial was designed to evaluate the safety and feasibility of HYP-RT with concomitant CDDP in LAHNC. Stage III and IV patients withnonmetastatic disease were enrolled. Patients were submitted to intensity modulatedradiation therapy, which comprised 55 Gy/20 fractions to the gross tumor and44-48 Gy/20 fractions to the areas of subclinical disease. Concomitant CDDPconsisted of 4 weekly cycles of $35 \mathrm{mg} / \mathrm{m} 2$. The primary endpoints were the treatment completion rate and acute toxicity.
\end{abstract}

Results: Twenty patients were enrolled from January 2015 to September 2016, and 12 (60\%) were classified as unresectable. All patients completed the total dose of radiotherapy, and 19 patients (95\%) received at least 3 of 4 cycles of chemotherapy. The median overall treatment time was 29 days (27-34). Grade 4 toxicity was reported twice (1 fatigue and 1 lymphopenia). The rates of grade 3 dermatitis and mucositis were 30\% and $40 \%$, respectively, with spontaneous resolution. Nasogastric tubes were offered to 15 patients (75\%) during treatment; 4 patients (20\%) needed feeding tubes after 2 months, and only 1 patient needed a feeding tube after 12 months.

Conclusion: HYP-RT with concomitant CDDP was considered feasible for LAHNC, and the rate of acute toxicity was comparable to that of standard concomitant chemoradiation. A feeding tube was necessary for most patients during treatment. Further investigation of this strategy is warranted.

Trial registration: ClinicalTrials, NCT03194061. Registered 21 Jun 2017 - Retrospectively registered.

Keywords: Head and neck neoplasm, Accelerated radiation therapy, Hypofractionated radiotherapy, Chemoradiotherapy, Locally advanced head and neck carcinoma (LAHNC), Concomitant chemotherapy

\footnotetext{
* Correspondence: jacintoaa@gmail.com

${ }^{1}$ Department of Radiation Oncology, Barretos Cancer Hospital, Rua Antenor

Duarte Villela, 1331, Barretos, SP 14.784-400, Brazil

Full list of author information is available at the end of the article
}

(c) The Author(s). 2018 Open Access This article is distributed under the terms of the Creative Commons Attribution 4.0 International License (http://creativecommons.org/licenses/by/4.0/), which permits unrestricted use, distribution, and reproduction in any medium, provided you give appropriate credit to the original author(s) and the source, provide a link to the Creative Commons license, and indicate if changes were made. The Creative Commons Public Domain Dedication waiver (http://creativecommons.org/publicdomain/zero/1.0/) applies to the data made available in this article, unless otherwise stated. 


\section{Background}

Concomitant chemoradiotherapy (cCRT) improves locoregional control (LRC) and overall survival (OS) in locally advanced head and neck cancer (LAHNC) compared with radiotherapy (RT) alone; consequently, chemoradiation is the standard of care for these patients [1]. Three-week $100 \mathrm{mg} / \mathrm{m}^{2}$ cisplatin concomitant with conventional fractionation radiotherapy (CFRT - 35 2-Gy fractions over 7 weeks) is the most studied regimen and is associated with significant toxicity, which compromises patient compliance and may not be suitable for all patients [2-5].

Altered fractionation is an alternative for patients who are not suitable for CCRT and can improve OS compared with CFRT alone [6, 7]. Accelerated RT, in which the total dose is delivered in a short period of time, has radiobiological advantages and is also associated with improved clinical outcomes $[8,9]$. Hypofractionation is an attractive method for accelerating RT and has been used with success with other tumor sites, showing comparable outcomes and a reduced cost compared to those of CFRT [10-13]. A remarkable moderate hypofractionated RT (HYP-RT) schedule for head and neck cancer, which delivers 55 Gy in 20 fractions (2.75 Gy per fraction) for 5 days per week, has been described in Birmingham/Edinburgh [14]. The biologically effective dose (BED) of the HYP-RT is approximately the same of CFRT [15]. The United Kingdom Head and Neck (UKHAN1) trial was one of the largest trial to demonstrate the superiority of cCRT over RT alone for LAHNC. In the UKHAN1 trial, almost $50 \%$ of patients were submitted to hypofractionated RT, including the HYP-RT schedule, and hypofractionation did not affect event-free survival compared with CFRT. The chemotherapy regimen used in the UKHAN1 trial was non-platin-based and, to the best of our knowledge, no data exists regarding HYP-RT concomitant with CDDP [16].

Patients from low- and middle-income countries (LMIC) have limited resources for RT and face long waiting times to be treated $[17,18]$. Consequently, in addition to the radiobiological and clinical benefits of accelerated RT, hypofractionation regimes can also be an important strategy to shorten treatment times and thus improve access to RT. Additionally, a short RT schedule is associated with better patient compliance [19].

The aim of this trial was to evaluate the feasibility and early safety of concomitant cisplatin in combination with HYP-RT in a high-volume center from an LMIC.

\section{Methods}

\section{Patients}

This open-label trial enrolled patients according to the following eligibility criteria: 1) biopsy-proven, non-metastatic, squamous cell carcinoma of the oropharynx, larynx and hypopharynx; 2) advanced stage disease, namely, stage III, IVa or IVb; 3) an ECOG performance status between 0 and 2;
4) aged from 18 to 70 years old; and 5) no history of previous malignancy. Each case was extensively discussed in multidisciplinary head and neck tumor board (HNTB) meetings to define the stage, resectability status and treatment recommendation. The HNTB comprised radiation oncologists, medical oncologists, radiologists and head and neck surgeons.

The study protocol was approved by the institutional review board and was conducted according to the Helsinki declaration. All patients provided specific written informed consent prior to participating in this trial. The study was also registered at ClinicalTrials.gov under the number NCT03194061.

\section{Treatment}

\section{Radiation therapy}

All patients were immobilized with a 5-point head-shoulder thermoplastic mask and subjected to a computed tomography $(\mathrm{CT})$ simulation with intravenous contrast. The primary tumor and gross nodal tumor were defined as the gross target volume (GTV). The clinical target volume of high risk (CTVhi) was an expansion of $0.5 \mathrm{~cm}$ from the GTV. Nodal neck delineation was based on a multi-institutional consensus guideline [20]. All regions deemed to be at high risk of subclinical disease were delineated by the physician as intermediate risk and named CTVInt. Nodal regions with a low risk of harboring subclinical disease were defined as CTVlow. Another tridimensional $0.5-\mathrm{cm}$ expansion from each CTVs was performed to generate the respective planning target volume (PTV). The simultaneous integrated boost (SIB) intensity modulated radiation therapy (IMRT) technique was used for all cases once per day for 5 days per week. The total dose for PTVhi was 5500 cGy in 20 fractions of 275 cGy (RT 55 Gy). Assuming the $\alpha / \beta$ ratio to be $10 \mathrm{~Gy}$ for head and neck carcinoma, the estimated BED for HYP-RT is about $66 \mathrm{~Gy}_{10}$, which is almost equivalent to CFRT [15]. Areas of intermediate risk (PTVint) for subclinical disease received $4800 \mathrm{cGy}$, and areas of lower risk for subclinical disease (PTVlow) received 4400 cGy. To optimize the overall length of treatment, all radiotherapy started on a Monday. The treatment plans were normalized to deliver $100 \%$ of the prescribed dose to at least $95 \%$ of each PTV $\left(D_{95 \%}=100 \%\right)$. Additionally, no more than $10 \%$ of the PTVhi received a dose higher than 60.5 Gy $\left(\mathrm{V}_{60.5 \mathrm{~Gy}} \leq 10 \%\right)$, and no more than $1 \%$ of the PTV received $<90 \%$ of the dose $\left(D_{99 \%} \geq 90 \%\right)$.

\section{Chemotherapy}

Patients received intravenous cisplatin at a dose of $35 \mathrm{mg}$ per square meter on days 1, 8, 15 and 22, concomitant with radiotherapy. Pre-medications were performed according to the institutional protocol. No dose reduction was allowed. Chemotherapy was 
delayed up to 2 weeks in cases of grade 3 or 4 toxicity. Subsequent administration was allowed if toxicity declined to below grade 2 .

\section{Assessments}

A baseline assessment was performed with a medical history, physical examination, flexible video laryngoscopy, a video deglutogram, and CT scan of the head, neck and chest. Human papillomavirus positivity was tested in all oropharyngeal cases. p16 immunohistochemistry was performed on formalin-fixed paraffin-embedded tissue sections that were cut to $4-\mu \mathrm{m}$ thicknesses using the CINtec p16INK4A assay, according to the manufacturer's instructions (CINtec Histology Kit; Ventana Medical Systems, Tucson, AZ, USA).

All patients were evaluated and graded for evidence of developing treatment toxicity according to the National Cancer Institute-Common Terminology Criteria for Adverse Events (NCI-CTCAE) version 4.0, except for radiation mucositis, which was graded according to NCI-CTCAE version 3.0. Assessments of patient adverse events were performed weekly during and up to 1 month after treatment, every month up to 4 months and then every 3 months up to 2 years.

All responses were defined by the HNTB. Tumor response evaluations were performed after 8 weeks and 16 weeks of treatment. The response assessment was performed by physical examination, head \& neck CT and video laryngoscopy. Response was based on the Response Evaluation Criteria in Solid Tumors (RECIST), version 1.1. $\left[{ }^{18} \mathrm{~F}\right]$ fluorodeoxyglucose positron emission tomography/CT (FDG-PET/CT) was not available for all patients during the response evaluation.

\section{Support therapy}

A dedicated oncology dietician evaluated all patients at baseline, weekly during and up to 1 month after treatment, and every 1-3 months thereafter according to the patient needs. During each visit, the anthropometric parameters were collected; the body mass index (BMI) and percent weight loss were calculated, and dietary intake was estimated by 24-h recall. All patients received oral glutamine during $\mathrm{RT}$, and nutritional supplementation was promptly initiated for patients with minimal weight loss. Patients with weight loss higher than $5-10 \%$ of the initial body weight (defined as the weight measured before treatment) and patients with grade 3 dysphagia were offered nasogastric tubes (NGTs).

Prophylactic and therapeutic photobiomodulation (PBM) with a low-level laser for oral mucositis was offered to all patients during treatment. All patients had post-diagnosis follow-up visits with a speech therapist.

\section{Study design and statistical analysis}

The primary endpoint of the study was the completion rate of treatment. The treatment was considered completed for patients who received at least $90 \%$ of the radiation dose (49.5 Gy), with a cumulative cisplatin dose of $105 \mathrm{mg} / \mathrm{m}^{2}$ (at least 3 cycles) and with an overall treatment time (OTT) below 35 consecutive days.

Toxicity was another primary endpoint, and we determined that the treatment would be considered unsafe if the rate of grade 4 mucositis and dermatitis was higher than $15 \%$. No data exists on the use of concomitant CDDP concurrent with HYP-RT. In our previous investigation, the rate of patients who received at least 2 of 3 cycles of cCRT after induction chemotherapy was approximately $85 \%$ [21]. We expected that at least $75 \%$ of patients would complete the treatment with no significant delay and no severe acute toxicity. Therefore, we could conclude the feasibility of this protocol with $95 \%$ certainty if 15 of 20 patients completed the treatment as stated.

\section{Stopping rule}

The safety analysis of the HYP-RT and concurrent chemotherapy was performed in a three-steps process within the study. To evaluate the completion rate and acute toxicity, we defined that each patient had to have a minimal follow up of 3 months after the cCRT:

A) STEP 1: In the first step, 5 unresectable cases were included. The criteria for moving forward to STEP 2 was that at least 3 patients $(3 / 5-60 \%)$ could complete the treatment as described in Section "Study design and statistical analysis". If 3 or more patients had not completed the treatment then the study protocol would be deemed too toxic and the study would be re-evaluated with respect to the feasibility.

B) STEP 2: Five more patients - resectable or unresectable - were included. The criteria for moving to STEP 3 was that at least 7 patients (7/10-70\%) could complete the treatment as described in Section "Study design and statistical analysis". If 4 or more patients (among the first 10 patients) had not completed the treatment then the study protocol would be deemed too toxic and the study would be re-evaluated with respect to the feasibility.

C) STEP 3: Finally, after the confirmation of the protocol safety among the first 10 patients, the STEP 3 was commenced including 10 more patients up to a total of 20 cases. The study protocol would be considered feasible if at least 15 patients (15/20-75\%) could complete the treatment as described in Section "Study design and statistical 
analysis". If 6 or more patients had not completed the treatment then the study protocol would be deemed too toxic and not feasible.

Additionally, a dose-limiting toxicity (DLT - defined as any grade 4 toxicity) was established. Basead on standard cCRT arm of RTOG 0522, RTOG 9501 and our previous experience on LAHNC, the DLT estimated for this study was $25 \%$. After each STEP, If the rate of toxicity exceeded the estimated DLT or if at any time a Grade 5 (death) was registered, the accrual would be stopped and the protocol had to be evaluated by an independent data monitoring committee with respect to the feasibility of the study protocol.

\section{Results}

\section{Patient characteristics}

From January 2015 to September 2016, 20 patients were enrolled in this study. The accrual rate was mainly determined by the protocol stopping rule. Figure 1 presents a CONSORT diagram to demonstrate the patient flow in the protocol. The patient characteristics are listed on
Table 1 . The median age was 53-years (42-69), 16/20 (80\%) were male and 19/20 (95\%) had a history of smoking. Most patients had stage IV disease (75\%), T3/T4 (80\%) and N2/N3 (75\%). The disease was considered unresectable in $12 / 20$ patients $(60 \%)$, and there were no HPV-related tumors.

\section{Treatment compliance}

Treatments started up to 10 days after accrual, and all patients were treated per protocol. In the STEP 1 it all patients completed the treatment according to the protocol (Section "Study design and statistical analysis"), and the protocol was moved forward to the next STEP. In STEP 2, 70\% of completion was expected and $90 \%$ of patients completed the treatment. In STEP 3 It was expected that at least $75 \%$ of all 20 patients had completed cCRT. Nineteen of 20 patients (95\%) received at least 3 cycles of chemotherapy. All patients received the total dose of RT. The median treatment time was 29 days (27-34) and the length of treatment was greater than 30 days in 1 patient. The completion rate was 95\% then study protocol was considered feasible. The number of

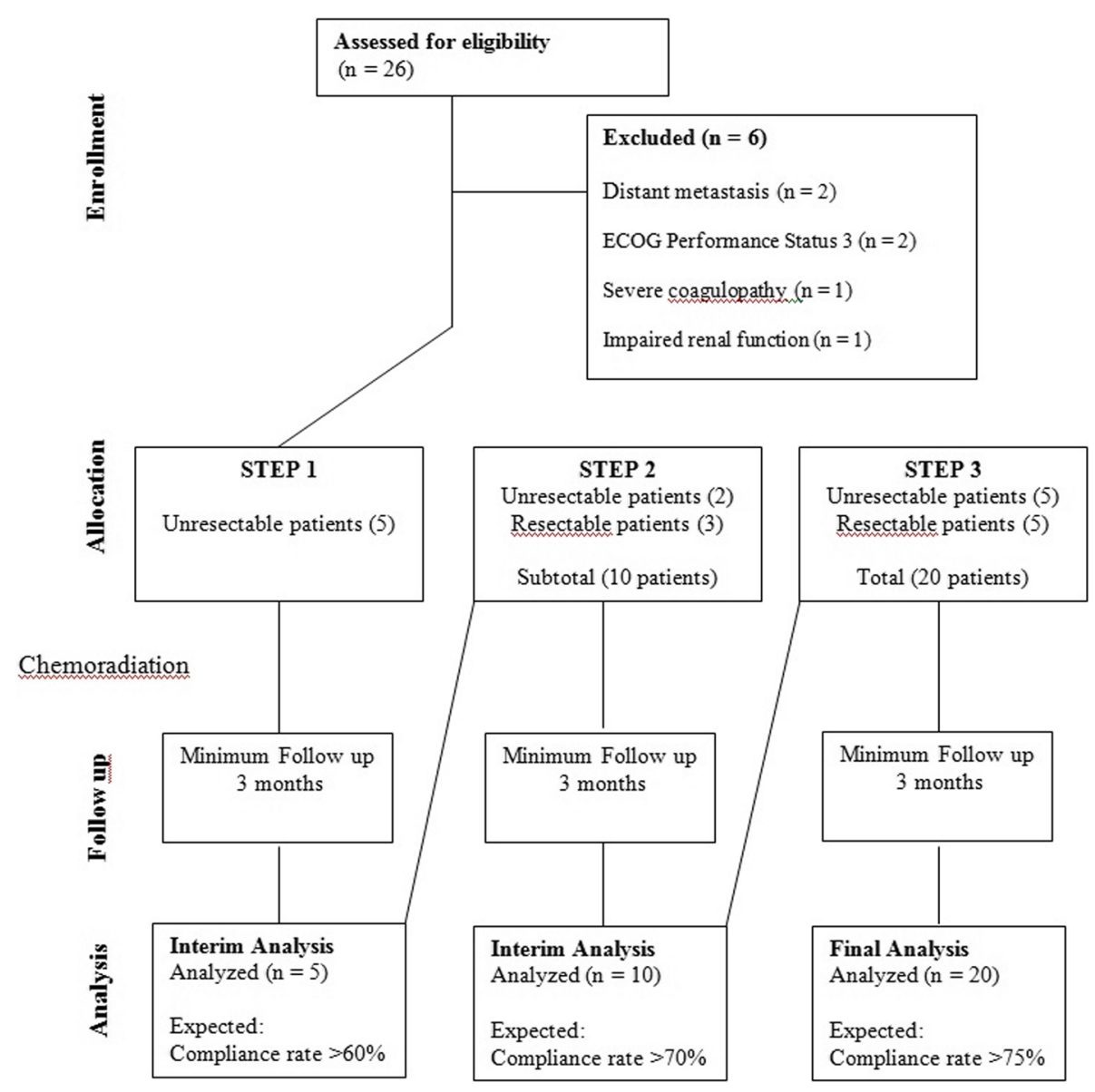

Fig. 1 CONSORT diagram showing the flow of participants through each steps of the feasibility trial 
Table 1 Patient characteristics

\begin{tabular}{|c|c|}
\hline \multirow{2}{*}{$\begin{array}{l}\text { Median age- } \\
\text { years (range) }\end{array}$} & $53(42-69)$ \\
\hline & Patients (\%) \\
\hline \multicolumn{2}{|l|}{ Gender } \\
\hline Male & $16(80)$ \\
\hline Female & $4(20)$ \\
\hline \multicolumn{2}{|l|}{ Primary site } \\
\hline Oropharynx & $10(50)$ \\
\hline Larynx & $6(30)$ \\
\hline Hypopharynx & $4(20)$ \\
\hline \multicolumn{2}{|c|}{ Performance status (ECOG) } \\
\hline 0 & $4(20)$ \\
\hline 1 & $14(70)$ \\
\hline 2 & $2(10)$ \\
\hline \multicolumn{2}{|l|}{ Clinical stage } \\
\hline III & $5(25)$ \\
\hline IV & $15(75)$ \\
\hline \multicolumn{2}{|l|}{ Tumor (T) - Stage } \\
\hline $\mathrm{T} 1$ & $1(05)$ \\
\hline $\mathrm{T} 2$ & $3(15)$ \\
\hline T3 & $8(40)$ \\
\hline T4 & $8(40)$ \\
\hline \multicolumn{2}{|l|}{ Nodal (N) - Stage } \\
\hline No & $5(25)$ \\
\hline N1 & $0(00)$ \\
\hline N2 & $11(55)$ \\
\hline N3 & $4(20)$ \\
\hline \multicolumn{2}{|l|}{ Resectability } \\
\hline Resectable & $8(40)$ \\
\hline Unresectable & $12(60)$ \\
\hline \multicolumn{2}{|l|}{ Smoking status } \\
\hline Current & 11 \\
\hline Former & 8 \\
\hline Never & 1 \\
\hline
\end{tabular}

patients who received 4, 3 and 2 chemotherapy cycles was 11,8 and 1 , respectively. Table 2 outlines the completion rate according to each step of the protocol.

\section{Toxicity}

No treatment-related deaths occurred. Only 2 patients (10\%) experienced grade 4 toxicity (fatigue and lymphopenia). No patient presented grade 3 neutropenia. The rate of grade $3 / 4$ lymphopenia was $20 \%$. Renal function was stable, and only 1 patient (5\%) showed a small creatinine elevation (grade 1) during treatment. Table 3 presents the most important toxicities observed during treatment.
Table 2 Feasibility criteria and completion rate according to each step of the trial. The results are presented according to each feasibility criterion (per treatment)

\begin{tabular}{|c|c|c|}
\hline & $\begin{array}{l}\text { Target } \\
\text { Range }\end{array}$ & $\begin{array}{l}\text { Delivered } \\
\text { Median (range) }\end{array}$ \\
\hline \multicolumn{3}{|l|}{ STEP 01 (5 unresectable patients) } \\
\hline Radiotherapy dose & 49.5-55 Gy & 55 Gy (55) \\
\hline Cisplatin dosage $\left(\mathrm{mg} / \mathrm{m}^{2}\right)$ & $105-140$ & $140(105-140)$ \\
\hline Overall treatment time (days) & $\leq 35$ & $29(29-30)$ \\
\hline \multicolumn{3}{|c|}{ STEP 02 (step $01+5$ patients) Resectable or unresectable } \\
\hline Radiotherapy dose & 50-55 Gy & $55(55)$ \\
\hline Cisplatin dosage $\left(\mathrm{mg} / \mathrm{m}^{2}\right)$ & $105-140$ & $140(70-140)$ \\
\hline Overall treatment time (days) & $\leq 35$ & $29(27-34)$ \\
\hline \multicolumn{3}{|c|}{ STEP 03 (step $2+10$ patients) Resectable or unresectable } \\
\hline Radiotherapy dose & 50-55 Gy & $55(55)$ \\
\hline Cisplatin dosage $\left(\mathrm{mg} / \mathrm{m}^{2}\right)$ & $105-140$ & $122(70-140)$ \\
\hline Overall treatment time (days) & $\leq 35$ & $29(27-34)$ \\
\hline
\end{tabular}

No grade 4 dermatitis or mucositis occurred. The rate of grade 3 dermatitis was $30 \%$, and dermatitis was more frequent during the first week after treatment. Eight patients (40\%) experienced grade 3 mucositis during the last week of treatment. As outlined in Fig. 2, all patients had complete resolution of the mucositis and dermatitis up to 1 month after treatment.

Most patients (95\%) lost weight during treatment, and the median percentage of weight loss during treatment was $7.8 \%$. All patients received nutritional supplementation during treatment, and 15 patients (75\%) required NGT; most patients required NGTs due to weight loss > $5 \%$ and grade 3 dysphagia or odynophagia. At the last follow-up visit, only one patient was still using the NGT.

Table 3 Acute toxicity of concurrent cisplatin with hypofractionation (HYP-UK) for locally advanced head and neck cancer. According to the Common Terminology Criteria for Adverse Events Version 3.0

\begin{tabular}{llll}
\hline Acute adverse event & $\begin{array}{l}\text { Grade } 1 / 2 \\
\mathrm{n}(\%)\end{array}$ & $\begin{array}{l}\text { Grade 3 } \\
\mathrm{n}(\%)\end{array}$ & $\begin{array}{l}\text { Grade 4 } \\
\mathrm{n}(\%)\end{array}$ \\
\hline Hematological & $7(35)$ & 0 & 0 \\
Anemia & $7(35)$ & $3(15)$ & $1(5)$ \\
Lymphopenia & $1(5)$ & 0 & 0 \\
Thrombocytopenia & $5(25)$ & $1(5)$ & 0 \\
Neutropenia & 0 & $1(5)$ & 0 \\
Febrile Neutropenia & & $6(30)$ & 0 \\
Non-Hematological & $19(95)$ & $8(40)$ & 0 \\
Radiodermatitis & $15(75)$ & 0 & 0 \\
Mucositis & $1(5)$ & & \\
Increased serum creatinine & & & \\
\hline
\end{tabular}




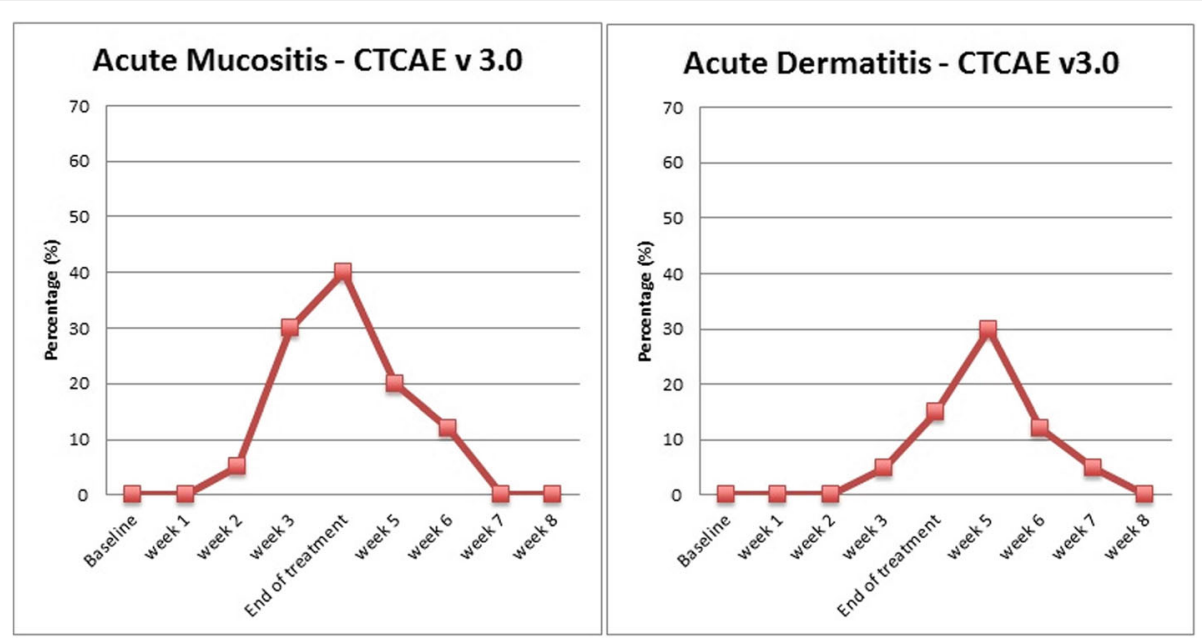

Fig. 2 Rate of mucosistis and radiation dermatitis from the treatment start (baseline). According to the Common Terminology Criteria for Adverse Event version 3.0

\section{Tumor response and salvage surgery}

The overall rate of response ( $\mathrm{T}$ and $\mathrm{N}$ ) was $95 \%$ after 2 months. The rate of complete response (CR) of the primary site (T) was $85 \%$. The nodal CR was $40 \%$. The overall CR was $85 \%$ for patients with resectable disease and 35\% for patients deemed unresectable.

\section{Discussion}

Altered fractionation is a well-established alternative of RT in the LAHNC treatment because many studies have demonstrated its superiority in disease control and survival compared with CFRT [7]. By reducing the OTT, the accelerated repopulation effect is minimized, which may explain the improved outcomes when treatment is accelerated $[8,9,15]$. Hypofractionation is a remarkable method for accelerating cancer treatment and is associated with better RT compliance [19]. Additionally, radiobiological and long-term clinical data have suggested that the HYP-RT regimen of $55 \mathrm{~Gy}$ in 20 fractions is, at least, equivalent to CFRT for LAHNC $[15,16]$. However, despite recent technological RT advances and successes in other tumor sites [10-12], the use of hypofractionation regimens with radical intent in LAHNC is modest and restricted to a few countries, particularly the United Kingdom [14, 16, 22-24]. The main reason for this restriction is the toxicity concern regarding the high dose per fraction, notably with concomitant chemotherapy [25]. Moreover, whether concomitant CDDP improve outcomes in the context of hypofractionation for LAHNC is unknown. The long-term outcomes of the UKHAN1 trial, which included CFRT and HYP-RT, demonstrated good compliance, a low rate of late toxicity, improved disease control, fewer new tumors and reduced mortality when cCRT was compared to RT alone [16]. Nevertheless, the chemotherapy used in the UKHAN1 trial was non-platin based. Although Madhava and colleagues have already demonstrated the feasibility of carboplatin with HYP-RT, to the best of our knowledge, our trial is the first to address the feasibility of concurrent CDDP with hypofractionation in LAHNC [14]. With a $95 \%$ of completion rate, our early data demonstrate the good compliance and suggest the feasibility of this protocol for patients from a middle-income country.

The standard concomitant chemotherapy with CFRT comprises the full dose of CDDP (3 cycles of $100 \mathrm{mg} / \mathrm{m}^{2}$ every 21 days), and the treatment is associated some toxicity, poor treatment compliance and treatment delays $[2,4,5]$. Indeed, standard cCRT may not be suitable for all patients $[1,3]$. In a Brazilian report of CRT for patients with unresectable, stage IV, non-metastatic disease, the full dose of CDDP was not feasible due to high treatment-related toxicity and unplanned treatment breaks [3]. Although we were concerned about severe toxicity, two patients only (10\%) experienced grade 4 toxicity during treatment (fatigue and lymphopenia) and the established DLT was not reached. In recent trials which the control arms was the standard cCRT, most IMRT based, the rate of grade 3-4 mucosistis, disfagia and neutropenia was $38-40 \%, 32-70 \%$, and $19-26 \%$, respectively [26-28]. Comparing with those IMRT based trials our rate of grade 3 neutropenia, mucositis and dermatitis was considered acceptable [26-28]. The standard CCRT in the control arm of RTOG 0129 was associated with high rate of enteral tube need $(70 \%)$ during treatment and the long term need was 36\% [26]. In the current trial NGT was offered to $75 \%$ of patients during treatment, but 4 patients $(20 \%)$ and 1 patient only (5\%) were still using NGT 1 month and 12 months after treatment, respectively.

The most important radiobiological advantage of hypofractionation involves its ability to enable explorations aimed towards reducing the OTT and, thereafter, minimizing the effect of accelerated repopulation of head and neck 
squamous cell carcinoma [15]. A median local control reduction of $1.4 \%$ per day of GAP and approximately $11 \%$ per week of OTT prolongation have been estimated [29, 30]. LAHNC tumor burden and treatment toxicity are associated with significant suffering and disability and are the primary causes of treatment interruptions [31]. Because this was a safety trial, we determined that the OTT was as important as the completion rate, which used the OTT as a parameter to define treatment feasibility. Indeed, we were concerned that by increasing acute toxicity, the OTT would be prolonged due to unplanned treatment breaks. However our data suggest the good tolerance profile of the protocol, whereby no patient needed a treatment break due to toxicity and no significant delay was experienced. In addition to treatment toxicity, many other factors underlie RT prolongation, including low socioeconomic status, a long treatment course, an unplanned equipment breakdown and the travel distance from the patient's home to the RT site [19]. An integrated multidisciplinary approach plays an essential role in improving tolerance and RT compliance in LAHNC $[31,32]$. In our institution, all head and neck patients receive continuous assistance of a social worker, and they are followed by a well-structured supportive care team during treatment and follow-up. All patients in the protocol were offered laser therapy for the normal mucosa, oral glutamine, early nutritional interventions and speech therapy during and after treatment. We believe that our integrated multidisciplinary care had a positive impact on treatment compliance, and a limitation of our outcomes may be that they are not representative of many centers from LMIC which cannot provide good integrated supportive care for patients with LAHNC OS and disease-free survival are the main endpoints for assessing the effectiveness of conservative treatment for LAHNC; however, the tumor response rate may be a surrogate of long-term LRC and survival, although this has not been reported in most prospective trials [33]. Despite the advanced tumor stages in our population the overall tumor response in our trial was consistent with the literature and with our previous experience with LAHNC [21].

According to the International Atomic Energy Agency (IAEA), an estimated $50 \%$ or more of RT patients will not have access to treatment in LMIC. Again, recent improvements in RT delivery have allowed the safe use of hypofractionation in many other cancer sites, and short-course RT is associated with cost effectiveness, patient convenience and better compliance [10-12, 17-19]. Thus, HYP-RT for LAHNC may be an important approach towards improving RT resource sparing. The design, short follow-up time and small patient number of this single institutional feasibility trial does not allow a strong conclusion about long-term outcomes; however, these findings must be further explored in large prospective clinical trials.
Currently, the IAEA is conducting a phase 3 trial, known as the HYPNO-trial (NCT02765503), to compare hypofractionation with accelerated normo-fractionated RT for LAHNC. Another prospective trial from the University of Birmingham, the COMPARE trial (EudraCT No: 2014003389-26), is now comparing standard cCRT with hypofractionated RT with concomitant CDDP. Both studies are currently active and open for recruitment.

\section{Conclusion}

In summary, treatment of LAHNC with HYP-RT concurrent with cisplatin appears feasible and safe and is associated with a good response rate. These data highlight the potential usefulness of hypofractionation for LAHNC, especially for LMIC, where access to RT is poor. Long-term outcome data from the HYPNO and COMPARE trials are expected to provide definitive conclusions about HYP-RT for LAHNC.

\section{Acknowledgements}

We acknowledge the support of the Research Department (NAP - Núcleo de Apoio ao Pesquisador) of the Barretos Cancer Hospital and the assistance provided by all staff and residents of the Department of Radiation Oncology and the Department of Head and Neck Surgery, Barretos Cancer Hospital. Aditional acknowledgements to Dr. Marcos Duarte de Mattos and Dr. Diogo Dias do Prado, Radiation Oncologists of Barretos Cancer Hospital, Jose Giroldo Junior and Ana Carolina Queiroz Porto from the Multidisciplynary team of Head \& Neck department of Barretos Cancer Hospital.

\section{Funding}

The study protocol entitled "Feasibility of concomitant cisplatin with hypofractionated radiotherapy for locally advanced head and neck squamous cell carcinoma" was integrally sponsored by Barretos Cancer Hospital. The sponsor had no role in the study design and collection and analysis, interpretation of the data, decision to publish, or writing the manuscript.

\section{Availability of data and materials}

The datasets generated and/or analysed during the current study are not publicly available due to ethical restriction [them containing information that could compromise research participant privacy/consent] but are available from the corresponding author on reasonable request.

\section{Authors' contributions}

AAJ was the principal investigator and was a major contributor in writing the paper; AAJ and ESBF were responsible for radiotherapy treatments; LAR was the medical physicist responsible for the radiation planning; LSV, PM, JMD and GDJP were the medical oncologist of the participant patients; ALC, RRG, RCC, DBJ and CR were head and neck surgeon of the team and were responsible for staging, biopsy and surgery procedures;" HPC and RC were the research coordinators of the protocol; EZ and SMB were international investigators and reviewer of the protocol and manuscript. All authors read and approved the final manuscript.

\section{Ethics approval and consent to participate}

The study entitled "Feasibility of concomitant cisplatin with hypofractionated radiotherapy for locally advanced head and neck squamous cell carcinoma" was conducted according to the Helsinky declaration. The protocol was approved by the Internal Review Board of the Barretos Cancer Hospital/ Fundação PIO XII and it is registered inthe Brazilian Platform of Ethics in Research (http://plataformabrasil.saude.gov.br/visao/publico/indexPublico.jsf) under the registration Number CAAE: 32147014.4.0000.5437. All patients provided specific written informed consent prior to participating in this trial. The study was also registered at ClinicalTrials.gov under the number NCT03194061. 


\section{Consent for publication}

Not applicable

\section{Competing interests}

The authors declare that they have no competing interests.

\section{Publisher's Note}

Springer Nature remains neutral with regard to jurisdictional claims in published maps and institutional affiliations.

\section{Author details}

'Department of Radiation Oncology, Barretos Cancer Hospital, Rua Antenor Duarte Villela, 1331, Barretos, SP 14.784-400, Brazil. ${ }^{2}$ Department of Medical Oncology, Barretos Cancer Hospital, Rua Antenor Duarte Villela, 1331, Barretos, SP 14.784-400, Brazil. ${ }^{3}$ Department of Head and Neck, Barretos Cancer Hospital, Rua Antenor Duarte Villela, 1331, Barretos, SP 14.784-400, Brazil. ${ }^{4}$ International Atomic of Energy Agency - Vienna International Centre, PO Box 100, A-1400 Vienna, Austria. ${ }^{5}$ University of Maryland School of Medicine, 655 W. Baltimore Street, Baltimore, MD 21201, USA

\section{Received: 21 January 2018 Accepted: 3 October 2018}

Published online: 23 October 2018

\section{References}

1. Pignon JP, le Maitre A, Maillard E, Bourhis J, MACH-NC Collaborative Group. Meta-analysis of chemotherapy in head and neck cancer (MACH-NC): an update on 93 randomised trials and 17,346 patients. Radiother Oncol. 2009;92(1):4-14.

2. Adelstein DJ, Li Y, Adams GL, Wagner H Jr, Kish JA, Ensley JF, Schuller $D E$, Forastiere AA. An intergroup phase III comparison of standard radiation therapy and two schedules of concurrent chemoradiotherapy in patients with unresectable squamous cell head and neck cancer. J Clin Oncol. 2003;21(1):92-8

3. de Castro G Jr, Snitcovsky IM, Gebrim EM, Leitao GM, Nadalin W, Ferraz AR, Federico $\mathrm{MH}$. High-dose cisplatin concurrent to conventionally delivered radiotherapy is associated with unacceptable toxicity in unresectable, nonmetastatic stage IV head and neck squamous cell carcinoma. Eur Arch Otorhinolaryngol. 2007;264(12):1475-82.

4. Forastiere AA. Cisplatin and radiotherapy in the management of locally advanced head and neck cancer. Int J Radiat Oncol Biol Phys. 1993;27(2):465-70.

5. Forastiere AA, Goepfert H, Maor M, Pajak TF, Weber R, Morrison W, Glisson B, Trotti A, Ridge JA, Chao C, et al. Concurrent chemotherapy and radiotherapy for organ preservation in advanced laryngeal cancer. $N$ Engl Med. 2003:349(22):2091-8.

6. Bourhis J, Overgaard J, Audry H, Ang KK, Saunders M, Bernier J, Horiot JC, Le Maitre A, Pajak TF, Poulsen MG, et al. Hyperfractionated or accelerated radiotherapy in head and neck cancer: a meta-analysis. Lancet. 2006:368(9538):843-54.

7. Lacas B, Bourhis J, Overgaard J, Zhang Q, Gregoire V, Nankivell M, Zackrisson B, Szutkowski Z, Suwinski R, Poulsen M, et al. Role of radiotherapy fractionation in head and neck cancers (MARCH): an updated meta-analysis. Lancet Oncol. 2017:18(9):1221-37.

8. Overgaard J, Hansen HS, Specht L, Overgaard M, Grau C, Andersen E, Bentzen J, Bastholt L, Hansen O, Johansen J, et al. Five compared with six fractions per week of conventional radiotherapy of squamous-cell carcinoma of head and neck: DAHANCA 6 and 7 randomised controlled trial. Lancet. 2003;362(9388):933-40.

9. Overgaard J, Mohanti BK, Begum N, Ali R, Agarwal JP, Kuddu M, Bhasker S, Tatsuzaki H, Grau C. Five versus six fractions of radiotherapy per week for squamous-cell carcinoma of the head and neck (IAEA-ACC study): a randomised, multicentre trial. Lancet Oncol. 2010;11(6):553-60.

10. Start Trialists' Group, Bentzen SM, Agrawal RK, Aird EG, Barrett JM, Barrett-Lee PJ, Bliss JM, Brown J, Dewar JA, Dobbs HJ, et al. The UK standardisation of breast radiotherapy (START) trial a of radiotherapy hypofractionation for treatment of early breast cancer: a randomised trial. Lancet Oncol. 2008:9(4):331-41.

11. Start Trialists' Group, Bentzen SM, Agrawal RK, Aird EG, Barrett JM, BarrettLee PJ, Bentzen SM, Bliss JM, Brown J, Dewar JA, et al. The UK Standardisation of Breast Radiotherapy (START) trial B of radiotherapy hypofractionation for treatment of early breast cancer: a randomised trial. Lancet. 2008;371(9618):1098-107.
12. Erlandsson J, Holm T, Pettersson D, Berglund A, Cedermark B, Radu C, Johansson H, Machado M, Hjern F, Hallbook O, et al. Optimal fractionation of preoperative radiotherapy and timing to surgery for rectal cancer (Stockholm III): a multicentre, randomised, non-blinded, phase 3, non-inferiority trial. Lancet Oncol. 2017:18(3):336-46.

13. Sethukavalan P, Cheung P, Tang Cl, Quon H, Morton G, Nam R, Loblaw A. Patient costs associated with external beam radiotherapy treatment for localized prostate cancer: the benefits of hypofractionated over conventionally fractionated radiotherapy. Can J Urol. 2012;19(2):6165-9.

14. Madhava K, Hartley A, Wake M, Watkinson JC, Glaholm J. Carboplatin and hypofractionated accelerated radiotherapy: a dose escalation study of an outpatient chemoradiation schedule for squamous cell carcinoma of the head and neck. Clin Oncol (R Coll Radiol). 2006;18(1):77-81.

15. Fowler JF. Is there an optimum overall time for head and neck radiotherapy? A review, with new modelling. Clin Oncol ( $R$ Coll Radiol). 2007:19(1):8-22.

16. Tobias JS, Monson K, Gupta N, Macdougall H, Glaholm J, Hutchison I, Kadalayil L, Hackshaw A, Head UK, Neck Cancer Trialists Group. Chemoradiotherapy for locally advanced head and neck cancer: 10-year follow-up of the UK Head and Neck (UKHAN1) trial. Lancet Oncol. 2010;11(1):66-74.

17. Zubizarreta EH, Fidarova E, Healy B, Rosenblatt E. Need for radiotherapy in low and middle income countries - the silent crisis continues. Clin Oncol (R Coll Radiol). 2015;27(2):107-14.

18. Zubizarreta EH, Poitevin A, Levin CV. Overview of radiotherapy resources in Latin America: a survey by the International Atomic Energy Agency (IAEA). Radiother Oncol. 2004:73(1):97-100.

19. Ohri N, Rapkin BD, Guha D, Haynes-Lewis H, Guha C, Kalnicki S, Garg M. Predictors of radiation therapy noncompliance in an urban academic cancer center. Int J Radiat Oncol Biol Phys. 2015;91(1):232-8.

20. Gregoire V, Ang K, Budach W, Grau C, Hamoir M, Langendijk JA, Lee A, Le QT, Maingon $P$, Nutting $C$, et al. Delineation of the neck node levels for head and neck tumors: a 2013 update. DAHANCA, EORTC, HKNPCSG, NCIC CTG, NCRI, RTOG, TROG consensus guidelines. Radiother Oncol. 2014;110(1):172-81.

21. de Souza VL, de Aguiar Silva FC, Andrade Dos Anjos Jacome A, Calheiros Campelo Maia D, Duarte de Mattos M, Arthur Jacinto A, Elias Mamere A, Boldrini Junior D, de Castro CR, Roberto Santos C, et al. Efficacy and safety of a cisplatin and paclitaxel induction regimen followed by chemoradiotherapy for patients with locally advanced head and neck squamous cell carcinoma. Head Neck. 2016;38(Suppl 1):E970-80.

22. Benghiat $H$, Sanghera $P$, Cashmore J, Hodson J, Mehanna $H$, Simmons R, Massey P, Sangha G, Bode C, Cooper P. Four week hypofractionated accelerated intensity modulated radiotherapy and synchronous carboplatin or cetuximab in biologically staged oropharyngeal carcinoma. Cancer Clin Oncol. 2014;3(2):1

23. Mehanna H, Wong WL, McConkey CC, Rahman JK, Robinson M, Hartley AG, Nutting C, Powell N, Al-Booz H, Robinson M, et al. PET-CT surveillance versus neck dissection in advanced head and neck cancer. N Engl J Med. 2016;374(15):1444-54.

24. Sanghera P, McConkey C, Ho KF, Glaholm J, Hartley A. Hypofractionated accelerated radiotherapy with concurrent chemotherapy for locally advanced squamous cell carcinoma of the head and neck. Int J Radiat Oncol Biol Phys. 2007;67(5):1342-51.

25. Chen AM, Hsu S, Meshman J, Chin R, Beron P, Abemayor E, St John M. Effect of daily fraction size on laryngoesophageal dysfunction after chemoradiation for squamous cell carcinomas of the larynx and hypopharynx. Head Neck. 2017;39(7):1322-6.

26. Ang KK, Harris J, Wheeler R, Weber R, Rosenthal DI, Nguyen-Tan PF, Westra $\mathrm{WH}$, Chung $\mathrm{CH}$, Jordan RC, Lu C, et al. Human papillomavirus and survival of patients with oropharyngeal cancer. N Engl J Med. 2010;363(1):24-35.

27. Ang KK, Zhang Q, Rosenthal DI, Nguyen-Tan PF, Sherman EJ, Weber RS, Galvin JM, Bonner JA, Harris J, El-Naggar AK, et al. Randomized phase III trial of concurrent accelerated radiation plus cisplatin with or without cetuximab for stage III to IV head and neck carcinoma: RTOG 0522. J Clin Oncol. 2014; 32(27):2940-50.

28. Siu LL, Waldron JN, Chen BE, Winquist E, Wright JR, Nabid A, Hay JH, Ringash J, Liu G, Johnson A, et al. Effect of standard radiotherapy with cisplatin vs accelerated radiotherapy with Panitumumab in Locoregionally advanced squamous cell head and neck carcinoma: a randomized clinical trial. JAMA Oncol. 2016. https://doi.org/10.1001/jamaoncol.2016.4510.

29. Fowler JF, Lindstrom MJ. Loss of local control with prolongation in radiotherapy. Int J Radiat Oncol Biol Phys. 1992;23(2):457-67. 
30. O'Connor P. The impact of missed fractions in head and neck radiotherapy and how they can be minimised. Radiography. 2013;19(4):343-6.

31. Vulpe H, Ellis J, Huang SH, Atenafu EG, Jang RW, Rodin G, Ringash J. The "rocky treatment course": identifying a high-risk subgroup of head and neck cancer patients for supportive interventions. Support Care Cancer. 2017; 25(3):719-27.

32. Ouwens MM, Hermens RR, Hulscher MM, Merkx MA, van den Hoogen FJ, Grol RP, Wollersheim HC, Marres HA. Impact of an integrated care program for patients with head and neck cancer on the quality of care. Head Neck. 2009:31(7):902-10.

33. Bataini JP, Jaulerry C, Brunin F, Ponvert D, Ghossein NA. Significance and therapeutic implications of tumor regression following radiotherapy in patients treated for squamous cell carcinoma of the oropharynx and pharyngolarynx. Head Neck. 1990;12(1):41-9.

Ready to submit your research? Choose BMC and benefit from:

- fast, convenient online submission

- thorough peer review by experienced researchers in your field

- rapid publication on acceptance

- support for research data, including large and complex data types

- gold Open Access which fosters wider collaboration and increased citations

- maximum visibility for your research: over $100 \mathrm{M}$ website views per year

At BMC, research is always in progress.

Learn more biomedcentral.com/submissions 